can be easily manipulated by applying a small bias voltage between tip and surface. We will show that similar manipulations with adatoms belonging to a Co dimer results in the switching of the magnetic interaction from ferromagnetic to antiferromagnetic and vice versa (Fig. 1).

This work was supported by the Russian Science Foundation, Grant No. 17-72-20041

1. F. Meier, L. Zhou, J. Wiebe, R.Wiesendanger, Science 320, 5872 (2008)

2. B. Kiraly, A. N. Rudenko, W. M. J. van Weerdenburg, D. Wegner, M. I. Katsnelson, A. A. Khajetoorians, Nat. Commun. 9, 3904 (2018).

\title{
FRACTAL MODELS IN ANALYSIS OF ENERGY TRANSFER PROCESSES AT INTERMOLECULAR INTERACTION
}

\author{
Baratova A.A. ${ }^{1}$, Baktybekov K.S. ${ }^{1}$ \\ ${ }^{1)}$ L.N. Gumilyov Eurasian National University, Nur-Sultan, Kazakhstan \\ E-mail: aa.baratova@yandex.kz
}

The correlated pairs fade away and spatially distributed structures form in the systems in which annihilation processes have a place. Efficiency of these processes on a porous surface depends on its structural characteristics (pore size, degree of homogeneity).

The correlated pairs fade away and spatially distributed structures form in the systems in which annihilation processes have a place. Efficiency of these processes on a porous surface depends on its structural characteristics (pore size, degree of homogeneity). Convenient way for gathering of information about modification of reagent distribution and cluster formation is registration of kinetics delay which is accompanied by triplet excitation annihilation at different temperatures on ratio of reagent concentrations. Method of modeling of bimolecular interactions between donor and acceptor of electron excitation energy in solids by making use of cellular automata is offered in this paper. Reagents distribution on the surface of solids and their evolution is the fundamental scientific interest, since this is an example of self-organized system. Method of multifractal analysis is used for determination of statistical parameters of investigation system. Further researches will allow predicting radiation stability of solids. Obtained researches show that faster annihilation kinetics is observed in matrices with chaotic distribution of reagents. Distribution in system will change from accidental when acceptor molecule very fast finds donor molecule and interacts with it to cluster at which with decrease of trap density trapping probability decreases and from cluster to uniform for which interaction kinetics is described by simple formally kinetic approaching. Conducted multifractal analysis of reagents distribution at different time pieces of kinetic dependences showed that clustering and regulation processes occur faster in higher degree of order matrices.At chaotic distribution of reagents order 
degree of matrix less than for matrixes with multifractal and cluster distributions. As a result of spatial division of reagents, clusters formation and their uniform model surface occupation degree of order increases and reaches some constant significance corresponding to surface clusters formation, fractal dimension of which in the course of time doesn't change. Time of stable cluster formation depends on reagents initial distribution character and peculiarities of molecular matrix structural organization at different time intervals. Average number of molecules in cluster and percolation probability (power of infinite cluster) are determined by connectedness connectivity of clusters molecules which specifies effective topology of system and critical concentration.

Observed changes of matrix structural organization with different degree of initial order at energy migration also can to explain on the basis of open systems thermodynamics. Thermodynamic conditions of fractal structures formation can determine from analysis of Rényi generalized fractal dimensions spectrum Dq(q) (figure ). The annihilation kinetics decreases at transition from chaotic to cluster distribution of donor molecules as a result of excitation localization in the range of cluster.

1. Mandelbrot B. The fractal geometry of nature- San Francisco: Freeman, (1982).

2. Bozhokin S.V., Parshin D.A. Fractals and multifractals, - M. - Izhevsk, (2001).

3. Vanag V. Study of spatially extended dynamical systems using probabilistic cellular automata, Phys. Usp., vol. 42, pp. 413-434, (1999). 